\title{
De la verve poétique : le Verlaine seconde manière de Cellulairement
}

\section{Gilles Negrello}

\section{(2) OpenEdition \\ 1 Journals}

\section{Édition électronique}

URL : https://journals.openedition.org/recherchestravaux/706

DOI : 10.4000/recherchestravaux.706

ISSN : 1969-6434

\section{Éditeur}

UGA Éditions/Université Grenoble Alpes

\section{Édition imprimée}

Date de publication : 15 décembre 2014

Pagination : 31-46

ISBN : 978-2-84310-291-2

ISSN : 0151-1874

\section{Référence électronique}

Gilles Negrello, « De la verve poétique : le Verlaine seconde manière de Cellulairement », Recherches \& Travaux [En ligne], 85 | 2014, mis en ligne le 15 juin 2016, consulté le 29 octobre 2021. URL : http:// journals.openedition.org/recherchestravaux/706 ; DOI : https://doi.org/10.4000/recherchestravaux. 706 
Gilles Negrello

Lycée Champollion (Grenoble)

\section{De la verve poétique : le Verlaine seconde manière de Cellulairement}

D'où vient qu'à mon insu, mariés à ma voix,

Les mots harmonieux s'enchaînent sous mes doigts, Et qu'en mètres brillants ma verve cadencée Comme un courant limpide emporte ma pensée?

LAMARTINE $^{\mathrm{I}}$

La réapparition du manuscrit de Cellulairement ${ }^{2}$ et sa publication en $2013^{3}$ permettent de revenir sur la question de l'évolution stylistique de Verlaine. Cellulairement, le "chaînon manquant ${ }^{4}$ » entre Romances sans paroles (I874) et Sagesse (1880), marque en effet une étape décisive dans l'histoire de la manière poétique verlainienne. Composé en prison en 1873 et 1874, mis en ordre et recopié pour être publié en 1875 , et enfin abandonné puis démembré au profit des recueils ultérieurs (principalement Sagesse, Jadis et Naguère et Parallèlement), Cellulairement correspond non seulement à une étape importante, mais à un moment clé, situé au centre même de l'œuvre en vers du poète. Olivier Bivort affirme que "ce tournant aura des répercussions dans toute l'œuvres"

I. A. de Lamartine, «Souvenir d'enfance, ou la vie cachée», Harmonies poétiques et religieuses (1830).

2. Lors d'une vente à Paris, le Is décembre 2004.

3. P. Verlaine, Cellulairement suivi de Mes prisons, éd. P. Brunel, avec le fac-similé du manuscrit original de Cellulairement, Paris, Gallimard, coll. «Poésie/Gallimard», 20I3. Ce manuscrit sera dans la suite désigné comme le manuscrit principal du recueil.

4. C'est le titre de la préface de J.-L. Steinmetz à son édition de Cellulairement aux éditions du Castor Astral, en I992.

5. Dans l'introduction de son édition critique : Verlaine, Romances sans paroles suivi de Cellulairement, Paris, Librairie générale française, coll. «Le Livre de poche Classiques», 2010 ( $2^{\mathrm{e}}$ éd. revue), p. 5 I. 
et considère que les derniers poèmes du recueil (notamment «Via dolorosa» et le «Final») marquent une "rupture poétique ${ }^{6}$ » qui préfigure l'orientation prise ensuite par l'œuvre verlainienne. Nous voudrions montrer que ce changement d'esthétique et de «système ${ }^{7}$ » d'écriture s'est produit au cours de la rédaction de Cellulairement; qu'il a été pensé et mis en scène par Verlaine dans la composition de ce recueil, et qu'il consiste en une conversion du poète à la verve poétique. Cette hypothèse permettra de réévaluer les poèmes de la seconde partie du recueil, qui sont quasi-unanimement sous-estimés par les critiques : nous voudrions rétablir la lisibilité de ces textes - en particulier des "récits diaboliques ${ }^{8}$ " et des premiers poèmes chrétiens que Verlaine compose au moment du retour à la religion de son enfance ${ }^{9}-$, en rétablissant ces textes dans les catégories esthétiques qui leur sont contemporaines, et démontrer que leur prétendue médiocrité vient du fait qu'on les a jugés selon une axiologie esthétique postérieure, celle du symbolisme, qui les a rendus incompréhensibles.

On affirme souvent - au mépris du témoignage de Verlaine lui-même dans Mes Prisons (I893) - que certains poèmes de Cellulairement, en particulier les quatre premiers "récits diaboliques» (datés de juillet et août I873) et "Almanach pour l'année passée» (daté de septembre 1873), auraient été écrits avant la fameuse "affaire de Bruxelles» qui a conduit Verlaine en prison. Jacques Robichez suppose que ces textes auraient été ébauchés au printemps I873 à Jéhonville, peut-être retravaillés ensuite à Londres (quand Verlaine y retourne avec Rimbaud, de fin mai à début juillet), et que la date indiquée dans le manuscrit de Cellulairement serait celle de leur mise au propre ${ }^{\mathrm{ro}}$. Rien n'accrédite cette

6. Ibid., p. 50.

7. Verlaine lui-même emploie ce mot pour présenter ses projets de poèmes, dans une lettre à Edmond Lepelletier du I6 mai I873 : «Les vers seront d'après un système auquel je vais arriver. » (Correspondance générale I [I857-I885], éd. M. Packenham, Paris, Fayard, 2005, p. 3I4.)

8. "C'est des récits plus ou moins diaboliques", écrit Verlaine à Lepelletier dans la lettre du 24-28 novembre 1873 (ibid., p. 357), en parlant de quatre de ces textes : "Crimen amoris", "La Grâce», «Don Juan pipé» et "L’impénitence finale». Dans le recueil manuscrit, le premier de ces poèmes est daté d'août I873; les trois suivants de septembre I873. Le cinquième, "Amoureuse du diable», sera achevé ultérieurement : il est daté d'août 1874. L'ensemble, précédé d'un poème liminaire, constituera la section «Naguère» de Jadis et Naguère (1884).

9. C'est G. Goffette dans L’Autre Verlaine (Paris, Gallimard, coll. «Folio», 20o8, p. 6o) qui propose d'employer une telle expression plutôt que celle de "conversion ", inappropriée à l'itinéraire spirituel de Verlaine qui, après avoir appris en mai 1874, du directeur de la prison de Mons, que le jugement en séparation réclamé par son épouse avait été prononcé par le tribunal, renoue avec la foi catholique et réintègre l'Église, en se confessant d'abord, puis en communiant, lors de la fête de l'Assomption, le Is août I874 - comme il le raconte dans Mes Prisons.

Io. Verlaine entre Rimbaud et Dieu. Des Romances sans paroles à Sagesse, Paris, SEDES, 1982, p. Ioo et suiv. J. Robichez est suivi en cela par J.-L. Steinmetz (ouvr. cité, p. Io). 
hypothèse, sinon l'étonnante rapidité d'exécution (en outre, dans les circonstances matérielles et morales difficiles des premiers temps de l'incarcération) que suppose leur datation dans le manuscrit principal. Tout l'argumentaire d'Antoine Fongaro dans son article "Notes sur la genèse de Cellulairement $t^{\mathrm{II}}$ " repose sur un comptage du nombre de vers supposément composés par Verlaine (si l'on se fie aux dates indiquées à la fin de chaque pièce dans le recueil manuscrit) lors des deux périodes de son incarcération : d'abord du io juillet au 24 octobre I873, à la prison des Petits-Carmes à Bruxelles, puis du 25 octobre I873 au I6 janvier I875 à la prison de Mons. Les totaux indiquent que Verlaine aurait d'abord composé un grand nombre de vers en peu de temps et dans des conditions pénibles (il se plaint alors dans ses lettres de ne pas arriver à travailler beaucoup); puis qu'il aurait ralenti sa production, revenant à un rythme d'écriture qui correspond davantage à ses habitudes antérieures.

La démonstration arithmétique peut paraître convaincante, quoiqu'elle ne repose que sur des éléments externes à l'œuvre (des informations issues d'enquêtes biographiques et des passages de la correspondance); mais elle ne tient pas face au contenu même des textes en question. En effet, les « récits diaboliques» ne font sens que si on les suppose écrits après le drame de Bruxelles. Leurs thèmes - l'emprisonnement («La Grâce»), le défi lancé à Dieu qui se solde par un échec ("Crimen amoris» et "Don Juan pipé»), la rédemption manquée ("L'impénitence finale») - font écho à la situation pénitentiaire de Verlaine et à sa culpabilité, prononcée par le tribunal et sans doute aussi intériorisée progressivement par le condamné. Ce serait vraiment une coïncidence étonnante que Verlaine ait composé plus tôt dans sa vie ${ }^{\mathrm{i} 2}$, et, de façon prémonitoire, des poèmes où il est question de crime passionnel, d'encellulement, de conscience tourmentée par le remords, de transgression et d'orgueil qui conduisent à une débâcle spirituelle... Il paraît plus naturel de supposer, en dépit de l'extraordinaire fécondité poétique que cela suppose (Verlaine aurait écrit plus de sept cents vers en trois mois, de juillet à septembre 1873), que ces textes ont été composés dans les premiers temps de la détention. Ces poèmes se présentent en effet, sous le voile de la fiction, comme un bilan de l'aventure passionnelle et métaphysique partagée avec Rimbaud. «Mis au point dans la solitude conseillère de la prison préventive, les centaines de vers des Récits diaboliques (et surtout des trois premiers : Crimen amoris, La Grâce et L'Impénitence finale) se résument en quelque sorte à un testament psychologique

II. Revue des sciences humaines, avril-juin I957, p. I65-I7I.

I2. À une date indéterminée: A. Fongaro parle, à la suite de Y.-G. Le Dantec, d'œuvres de jeunesse, «trahiss[ant] trop ouvertement l'influence de Gautier et de Musset» (ibid., p. I69). 
de la vie essentielle avec Rimbaud ", écrit Jacques-Henri Bornecque ${ }^{13}$. Il semble cohérent, au plan psychologique, d'admettre que Verlaine a éprouvé le besoin de dresser un tel bilan de sa liaison avec Rimbaud, une fois celle-ci terminée, dans les semaines qui ont suivi l'"affaire de Bruxelles".

Il n'y a pas de raison non plus - si l'on admet que Verlaine a pu écrire un grand nombre de vers dès le début de son incarcération - de situer la composition de l' «Almanach pour l'année passé» au printemps de i873. En effet, cet ensemble de quatre poèmes s'est d'abord intitulé Mon almanach $1874^{14}$. Cette date est incompréhensible si l'on suppose ces textes écrits en mai I873: si Verlaine avait écrit ces textes à ce moment-là, l'ensemble aurait dû s'intituler soit Mon almanach 1872 (dans la mesure où il recueille des souvenirs de l'année passée ${ }^{\text {Is }}$ ), soit Mon almanach I873, si l'on suppose que la date du titre se rapporte au moment de l'écriture des poèmes. Mais, en fait, un almanach est prospectif ${ }^{6}$ : on le publie, à la fin d'une année, pour l'année suivante; il contient, outre le calendrier lunaire, des prévisions, notamment météorologiques, pour les douze mois à venir. Quel sens cela aurait-il eu pour Verlaine, libre, séjournant chez sa tante au Luxembourg belge en mai 1873, de se projeter vers l'année suivante? Il vaut mieux admettre que la date qui figure à la fin de ces textes ( $7^{\text {bre }} \mathrm{I} 873$ ») est bien celle de leur composition. En effet, il semble logique que, peu après sa condamnation à deux ans de prison (prononcée le 8 août), alors qu'il s'apprête à passer toute l'année I874 derrière les barreaux, Verlaine compose cet imagier des quatre saisons, qui ornera en quelque sorte sa cellule et lui donnera à voir des images, puisées dans ses souvenirs récents, du monde extérieur et de la liberté : dans le premier poème, une promenade dans la campagne ardennaise; dans le second, une halte dans une auberge; dans le troisième, l'ivresse associée à la saison des vendanges; dans le dernier, la ville de Londres. Pour le prisonnier qui ne connaît qu'une succession de jours indifférenciés ${ }^{17}$, l'almanach sera un moyen de se repérer dans le temps en se souvenant des sensations qui caractérisent le printemps, l'été, etc.

13. Verlaine, Paris, Seuil, coll. «Écrivains de toujours», 1966, p. I07.

I4. Lettre à Lepelletier écrite vers le 20 octobre I873, Correspondance générale I, ouvr. cité, p. 352 .

I5. La deuxième pièce de la série, "L'espoir luit...", évoque probablement l'échappée en Belgique, au cours de l'été précédent, avec Rimbaud, tandis que la quatrième, "Ah, vraiment, c'est triste...» rappelle l'hiver passé à Londres, et plus précisément la période où Verlaine y est resté seul, quand Rimbaud est revenu à Charleville, en décembre I872.

16. Le Grand Dictionnaire universel du XIX siècle de P. Larousse précise qu'almanach « s'emploie dans le sens de pronostic» et que l'expression «C'est un almanach de l'an passé» signifie que "c'est une chose qui n'est à présent d'aucune utilité».

I7. Voir le refrain de "Réversibilités» : "-Ah! dans ces tristes décors / Les Déjàs sont les Encors!» 
Nous proposons donc d'admettre l'hypothèse selon laquelle la plupart des dates qui figurent dans le manuscrit de Cellulairement ${ }^{18}$ correspondent effectivement à celles de la composition des poèmes, ce qui implique une écriture très rapide, un grand nombre de vers étant composés en quelques semaines ou quelques mois, surtout au début de la période d'incarcération. Si de nombreux spécialistes résistent à envisager cette possibilité, c'est que Verlaine a été jusqu'à cette date l'auteur d'une poésie rare, à l'écriture parcimonieuse. Mais précisément, nous allons maintenant voir que ce qui est au cœur même de la structure du recueil Cellulairement, c'est un changement d'esthétique : le renoncement à une ancienne forme d'écriture poétique, à la fois très dense et d'une venue nécessairement lente, et l'adoption d'un nouveau "système» d'écriture, permettant une production plus abondante, plus facile, mais aussi plus inégale.

Les poèmes du recueil Cellulairement sont tous datés, individuellement ou par groupe (dans le cas d' "Almanach pour l'année passée», de "Vieux Coppées» et du «Final»). Ils sont disposés, dans l'ensemble, par ordre chronologique, le long d'un intervalle de dix-huit mois compris entre le II juillet I873 (date $\mathrm{d}^{\prime}$ " Impression fausse», premier poème après le prologue «Au lecteur») et le I6 janvier $1875^{19}$. La première hypothèse d'interprétation de la structure du recueil consiste donc, naturellement, à y voir une chronique autobiographique - pas tout à fait au jour le jour, mais de mois en mois - du séjour du poète en prison. Mais la succession chronologique des dates des poèmes est interrompue, environ au milieu du volume, par un retour en arrière d'un an : après «Via dolorosa», daté de "Juin. Juillet I874", commence la série des poèmes narratifs : "Crimen amoris, vision", daté de "Juillet I873", puis «La Grâce, légende», "Don juan pipé, mystère" et "L'impénitence finale, chronique parisienne", tous trois datés "Brux. Août I873». Cette "anomalie» massive dans la suite chronologique des poèmes remet en question l'idée selon laquelle Cellulairement serait un memorandum de l'incarcération de Verlaine.

Cette première hypothèse semble toutefois pouvoir être maintenue au prix d'une légère reformulation : le recueil ne serait pas exactement le journal intime du poète emprisonné, mais plutôt la chronique de son retour à la religion. «Il [Verlaine] a tenu à tisser le fil, non seulement d'une chronologie de

I8. À deux exceptions près, dûment avérées et facilement explicables : les dates du prologue «Au lecteur» et du «Final» ont été modifiées par Verlaine.

19. La deuxième de ces deux dates, au moins, est fictive : sur le manuscrit, le «Final» est daté du 20 août I874; Verlaine a biffé cette date pour lui substituer celle de sa sortie de prison, afin que la chronologie du recueil coïncide avec la durée de son incarcération. 
son temps cellulaire, mais de son évolution intérieure», écrit Pierre Brunel ${ }^{20}$. Cette évolution conduit de la pièce liminaire, "Au lecteur", d'un ton tout profane, jusqu'au «Final», du plus ardent mysticisme. De plus, le livre se divise nettement en deux parties : la première, qui aboutit à "L'Art poëtique", est généralement considérée comme l'œuvre d'un poète athée, ou du moins agnostique; tandis que la seconde, formée par les cinq "récits diaboliques ${ }^{21}$ " encadrés par "Via dolorosa» et le "Final», est dominée par des préoccupations religieuses. Avec sa composition globalement chronologique et sa structure binaire, Cellulairement renverrait donc au modèle des Contemplations, recueil divisé en deux volets («Autrefois» et «Aujourd'hui»), et que Hugo présentait dans sa préface comme «les Mémoires d'une âme $e^{22}$ ». Mais cette lecture du recueil est sans doute faussée par la perspective a posteriori induite par sa publication posthume. L'existence de Cellulairement ayant été révélée par les travaux d'Ernest Dupuis au début du $\mathrm{xx}^{\mathrm{e}}$ siècle $^{23}$, le livre a été lu en fonction de la connaissance qu'on avait de la biographie du poète, principalement à travers son propre témoignage. Ainsi, d'après les souvenirs racontés dans Mes Prisons, "L’Art poëtique», daté d'avril I874, apparaît comme le dernier poème écrit avant la crise spirituelle qui conduira Verlaine à renouer avec la fréquentation des sacrements. Les mémoires du poète permettent aussi de savoir que le poème suivant, "Via dolorosa», intervient au terme d'une période consacrée à la lecture du Catéchisme de persévérance de Mgr Gaume et couronnée par une illumination devant une image lithographique du Sacré-Cour de Jésus. Mais, d'abord, les souvenirs de Verlaine sont sujets à caution : de l'étude d'un carnet de notes ayant appartenu au poète, Vernon Philip Underwood conclut :

C'est donc dès le début de son emprisonnement, et non pas, comme le dira le poète lui-même, à la nouvelle de la séparation légale obtenue par sa femme, qu'il commença à lire le livre de Mgr Gaume que lui fournit l'aumônier, à s’adonner aux lectures pieuses que ces pages du carnet indiquent, et même à composer des vers sous ces influences ${ }^{24}$.

20. Cellulairement suivi de Mes Prisons, éd. P. Brunel, ouvr. cité, p. 353.

2I. Malgré cette appellation, ces historiettes sont nettement édifiantes et même parfaitement orthodoxes, la victoire de Dieu sur les ruses de Satan y étant systématiquement célébrée.

22. V. Hugo, Les Contemplations, Paris, Gallimard, coll. «Poésie/Gallimard», I973, p. 27.

23. E. Dupuis, «L'évolution poétique de Paul Verlaine. À propos d'un manuscrit du poète», Revue des deux mondes, I ${ }^{\text {er }}$ décembre 1912, p. 595-632; repris dans Poètes et critiques, Paris, Hachette, I9I3, p. 197-280; "Étude critique sur le texte d'un manuscrit de P. Verlaine», Revue d'histoire littéraire de la France, juillet-septembre 1913, p. 498-516.

24. "Le cahier personnel de Verlaine», Revue des Sciences humaines, I955, p. 22I. 
Ensuite, si le recueil avait pu être publié en I875, sans nom d'auteur, comme le souhaitait Verlaine ${ }^{25}$, les lecteurs d'alors, ignorant les détails de l'histoire personnelle de l'auteur, auraient perçu le livre comme émanant, du début à la fin, d'une inspiration chrétienne. En effet, de nombreuses notations, dans la première partie du recueil, lui confèrent une tonalité d'emblée religieuse : citons, entre autres, le titre du poème "Réversibilités», qui renvoie au dogme de la Communion des Saints, interprété dans un sens expiatoire par Joseph de Maistre; les derniers vers de "La Chanson de Gaspard Hauser" ("Ô vous tous, ma peine est profonde : / Priez pour le pauvre Gaspard!»); ou encore cette déclaration de résignation chrétienne dans «Vieux Coppées VIII" : "Ô Belgique qui m’as valu ce dur loisir, / Merci! j'ai pu du moins réfléchir, et saisir, / [...] Les raisons de mon être immortel et divin». Envisagé indépendamment de toute documentation biographique, Cellulairement apparaît de bout en bout comme le témoignage chrétien d'un pécheur touché par la grâce alors qu'il se trouve en prison.

Le plan binaire du recueil, divisé selon un axe central marqué par "L'Art poëtique», ne correspond donc pas à "avant/après la conversion». La dualité très visiblement mise en scène par cette composition n'est pas d'ordre autobiographique ou spirituel, mais d'ordre esthétique : ce sont deux poétiques nettement distinctes qui s'opposent entre la première et la seconde partie - d'où la position centrale de "L’Art poëtique», qui donne la clé de la structure du recueil. Dans la première partie, se trouvent des poèmes brefs, utilisant majoritairement des mètres courts et une division strophique qui ménage beaucoup de blanc autour des textes. La seconde partie du recueil réunit au contraire des poèmes étendus (d'une centaine de vers ou plus), utilisant principalement des mètres longs (alexandrins et hendécasyllabes) et souvent sans division strophique. Les "Vieux Coppées», situés juste avant la césure marquée par "L'Art poëtique», jouent un rôle de transition entre ces deux parties et contribuent à l'équilibre binaire du recueil, en constituant à la fin de la première partie un ensemble de dix dizains, auquel répond symétriquement le «Final», composé de dix sonnets, qui clôt la seconde partie. Sur le fac-similé du manuscrit, on observe sur le premier feuillet, sous le titre Cellulairement et avant le poème liminaire "Au lecteur», un I. en chiffre romain écrit au centre de la page. On peut faire l'hypothèse qu'à ce numéro devait correspondre un II., qui se serait trouvé après "L'Art poëtique» si le manuscrit n'avait pas été découpé à cet endroit ${ }^{26}$. L'examen du manuscrit montre aussi que Verlaine avait initialement

25. Voir la lettre à Ernest Delahaye du 26 octobre 1875, Correspondance générale I, ouvr. cité, p. 443 .

26. Il manque, avant "Via dolorosa» le bas du feuillet 3I et les feuillets 32, 33 et 34 . 
prévu une présentation très compacte pour les poèmes de la seconde partie, et qu'il a rajouté ensuite sur le manuscrit des signes en forme d'équerre, pour indiquer aux protes d'y introduire des lignes blanches. Sans ces ajouts ${ }^{27}$, la dualité du recueil aurait été encore plus marquée visuellement par le contraste entre un premier versant aéré, entouré de beaucoup de blanc, et un second versant d'aspect plus massif, aux pages entièrement noircies.

Le recueil Cellulairement est donc composé en diptyque, de part et d'autre de "L'Art poëtique», qui représente la charnière centrale de l'ensemble. La première partie du recueil correspond à l'ancienne manière de Verlaine, caractérisée par une écriture poétique retenue et concentrée, aux formes grêles et sporadiques. La seconde partie du recueil illustre la nouvelle manière de Verlaine, associée thématiquement à son retour à la religion catholique, et caractérisée par d'amples et denses coulées verbales. La différence entre les deux manières de Verlaine dans Cellulairement ne tient pas seulement à leurs caractéristiques formelles opposées : elle découle d'un parti pris autant existentiel qu'esthétique.

Les poèmes situés avant «L'Art poëtique» (qui constitue un bilan de la période de la création verlainienne embrassant l'époque des Romances sans paroles et le début de l'emprisonnement) illustrent la première manière du poète, que caractérise subtilement Huysmans dans la présentation qu'il donne de Verlaine dans $A$ rebours:

Seul, il avait pu laisser deviner des au-delà troublants d'âme, des chuchotements si bas de pensées, des aveux si murmurés, si interrompus, que l'oreille qui les percevait, demeurait hésitante, coulant à l'âme des langueurs avivées par le mystère de ce souffle plus deviné que senti ${ }^{28}$.

Cette première manière de Verlaine est caractérisée par la brièveté : ses poèmes s'apparentent à des haïkus, parce qu'ils s'alimentent, non à la source verbale de l'imaginaire ${ }^{29}$, mais au contact du réel rencontré dans sa présence immédiate, dans son être-là. Cela apparaît en particulier dans la série formée

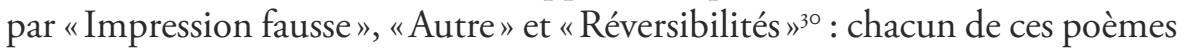

27. À titre d'exemple, dix-huit alinéas ont été insérés dans "L'impénitence finale», texte écrit d'abord d'un seul tenant.

28. Huysmans, À rebours, Paris, Flammarion, coll. «GF-Flammarion», p. 2 I4.

29. De ce point de vue, parmi les textes de la première partie du recueil, «Images d'un sou» doit être mis à part : c'est d'ailleurs le plus long de ces poème (66 vers) et le seul qui ne soit pas divisé en strophes.

30. Dans Cellulairement, le dernier poème est dissocié des deux autres, mais ils forment bel et bien un ensemble cohérent, comme cela apparaît dans Parallèlement, où ces textes sont les deuxième, troisième et quatrième de la série «Révérence parler». 
enregistre avec acuité les impressions produites par l'environnement carcéral sur une sensibilité qui n’y est pas encore habituée.

Les poèmes situés après "L'Art poëtique» relèvent d'un autre type d'écriture : ils se distinguent nettement des précédents, non seulement par leur format et leur versification ${ }^{31}$, mais surtout par la manière dont leur discours s'articule au réel. Ce n'est plus une poésie de l'instant, de l'illumination, mais une poésie du déploiement dans le temps, du processus. Les poèmes de la "conversion », «Via dolorosa» et le «Final», déroulent ainsi, de strophe en strophe pour le premier, d'un sonnet à l'autre pour le second, une durée qui est celle d'une évolution intérieure, avec ses étapes, ses arrêts et ses reprises. Chacune de ces deux pièces se termine sur un point d'orgue, qui correspond au consentement finalement donné par l'âme réticente à l'action en elle de la grâce divine. En outre, tous les poèmes de la seconde partie sont des textes à personnages. Tantôt le poète se met lui-même en scène dans les rôles, fixés par la tradition littéraire et religieuse, du pécheur repenti ou du mystique. Il devient alors un personnage de théâtre, qui entre dans un dialogue avec le Christ : la fin de "Via dolorosa ${ }^{32}$ » et l'ensemble $\mathrm{du}$ «Final» adoptent ainsi une forme dialoguée, usant du tiret long initial pour marquer chaque changement d'interlocuteur. Tantôt les poèmes prennent la forme de récits narrant l'histoire de personnages fictifs. Mais il est à noter que les héros et héroïnes des "récits diaboliques» sont peut-être encore des masques du poète lui-même. L'héroïne de «La Grâce», enfermée dans un cachot avec la tête de son mari (qu'elle a assassiné), dont le crâne est habité par un démon qui essaye de la damner, est un double de Verlaine lui-même, enfermé dans sa cellule, avec le souvenir de Rimbaud (qu'il a voulu assassiner ${ }^{33}$ ), souvenir obsédant comme un spectre qui essaierait de le ramener vers le passé, quand lui aspire à se réconcilier, par le repentir, avec Dieu. D’ailleurs, la situation de ce poème narratif, «La Grâce», se retrouve quasi-identique, mais cette fois à la première personne du singulier, dans «Un pouacre», où le poète emprisonné reçoit la visite d'un fantôme importun, incarnant sa mauvaise conscience : «Avec les yeux d'une tête de mort / Que la lune encore décharne, / Tout mon

3I. On peut parler à leur propos de "versification prosaïque», caractérisée par le débordement du rythme syntaxique sur le patron métrique (avec un grand nombre d'enjambements, de rejets et de contre-rejets) et par des coupes erratiques qui multiplient les mesures irrégulières dans des alexandrins souvent sans césure.

32. À partir de la onzième strophe. Mais sur le manuscrit, la marge de gauche a été rognée quand les pages du cahier d'écolier utilisé par Verlaine pour y copier ses poèmes ont été découpées afin d'être montées sur vélin et reliées, de telle sorte que les tirets longs et les guillemets qui explicitaient le système dialogique du texte ont disparu des douzième et treizième strophes.

33. C'est du moins pour «tentative d'assassinat» que Verlaine a été jugé et condamné à deux ans de prison. 
passé - disons tout mon remords / Ricane à travers ma lucarne.» Rimbaud, "l'époux infernal», semble avoir inspiré les héros de "Crimen amoris» et de "Don Juan pipé», qui se révoltent contre Dieu au nom de l'amour charnel et finissent punis. L'héroïne relapse de "L’impénitence finale», qui retombe dans l'adultère après avoir rencontré le Christ venu lui pardonner sa première faute, semble représenter le pressentiment des chutes futures du poète catholique repris par le péché. Enfin, le suborneur d' "Amoureuse du Diable», qui fait l'éloge de la soûlographie et maltraite sa compagne, n'est-ce pas Verlaine lui-même tel qu'il s'est comporté avec Mathilde? Ce poème se termine par un vers que sans nul doute Verlaine, du fond de sa solitude carcérale, prend à son propre compte : «Elle ne savait pas que l'Enfer, c'est l'absence!» Ainsi, en adoptant une organisation narrative, rendue possible par leur longueur, les poèmes de la seconde partie de Cellulairement versent l'inspiration autobiographique dans le domaine de l'imaginaire.

L'opposition des deux manières de Verlaine n'est pas uniquement de nature formelle, mais repose sur l'articulation de la vie et de l'œuvre. L'esthétique de «la chanson grise» correspond à une pratique de l'écriture poétique qui se glisse dans les interstices d'un quotidien intensément vécu. La création est alors seconde par rapport à l'expérience éprouvée : elle s’insère dans les pauses contemplatives d'une existence mouvementée, afin d'en fixer les impressions les plus intenses. Ce rapport particulier de l'écriture à la vie culmine à l'époque de la liaison avec Rimbaud et des Romances sans paroles. Mais à partir du moment où il est emprisonné, Verlaine va rapidement se trouver sevré de mouvement et de sensations (notamment celles de l'ivresse, procurée par la chair ou par l'alcool). La poésie devient alors une occupation qui permet de combler ce vide (d'où la composition de pièces d'envergure, nécessitant un travail suivi), en recourant à l'imagination - ce qui explique la place des poèmes narratifs dans la seconde partie du recueil. Cette hypothèse explique pourquoi le passage d'une manière à la suivante ne coïncide pas avec l'entrée de Verlaine en prison, mais se produit en différé : au début de son incarcération, le poète est encore dans l'expérimentation de ce qui lui arrive, dans la découverte de sensations nouvelles. Ceci reste valable jusqu'à "Réversibilités», écrit peu après son transfert à la prison de Mons en octobre I873. "Images d'un sou» marque la fin de cette captation de l'environnement, remplacé par une fuite dans l'imaginaire. Dans les mois qui suivent, le prisonnier s'installe dans une routine que suggère la datation finale des "Vieux Coppées» : «Mons - I874, Janvier, Février, Mars et passim». Le traintrain monotone de la prison rend le réel désert et le quotidien vide; dès lors l'écriture devient un palliatif et l'imagination une échappatoire, ce qui détermine le basculement de Verlaine 
vers une seconde manière, caractérisée par une écriture relâchée et abondante, où prévaut la narration.

Le passage de la première à la seconde manière de Verlaine, dans Cellulairement, donne inévitablement au lecteur d'aujourd'hui l'impression d'une "chute de ton ${ }^{34} »$ voire d'une perte de l'inspiration : les poèmes de la seconde partie paraissent lourdauds, prosaïques et fastidieux. Pourtant, la mésestime dans laquelle sont largement tenus ces textes est sans doute injustifiée : que Verlaine lui-même fût attaché à cette partie de son œuvre ${ }^{35}$ n'est peut-être pas un argument suffisant en faveur de sa qualité; mais peut-on penser que Rimbaud se fût donné la peine de recopier trois de ces poèmes ${ }^{36} s^{3}$ il les avait jugés médiocres? Nous voudrions montrer que le discrédit esthétique du Verlaine seconde manière repose sur une erreur de perspective qui fausse le regard porté a posteriori sur ces textes et que la notion de «verve poétique», telle qu'elle a cours à l'époque, permet de corriger cette erreur de jugement.

La définition du mot "verve» dans Le Grand Dictionnaire universel $d u$ $X I X^{e}$ siècle de P. Larousse (Paris, I870, t. XV, p. 949) fait apparaître qu’à cette date, la notion de "verve" s'attache avant tout à la poésie. En effet, après des considérations étymologiques et une définition sémantique ("Chaleur d’imagination qui anime le poëte, l'artiste, le causeur, quand ils composent ou qu'ils parlent»), la première association de mots lexicalisée que mentionne l'article est : "Verve poétique», suivie par : "Être en Verve. Parler, écrire de Verve. Ce poète vieillit, il n'a plus de VERVE. C'est un peintre, un musicien qui manque tout à fait de verve [...]». Après avoir donné un deuxième sens du mot, jugé vieilli («Bizarrerie, caprice»), l'article développe une rubrique "Encyclopédie», située dans le domaine "Littérature», qui éclaire remarquablement le sens de ce qu’a tenté Verlaine dans la seconde partie de Cellulairement:

On considérait autrefois la verve poétique comme une sorte d'ivresse, une extase, quelque chose comme une vision céleste; on lui donnait pour source une influence divine. Ces fictions sont parties avec les Muses. Il est resté l'homme avec ses facultés intellectuelles. Quand le poète, l'artiste, se représente fortement l'objet qu'il veut peindre, le sentiment qu'il veut exprimer, et que son esprit s'échauffe, que son cœur s'émeut, il se trouve bientôt dans cet état particulier d'enthousiasme qui

34. Cellulairement, éd. O. Bivort, ouvr. cité, p. 47.

35. Voir la lettre du 29 août I887 dans Lettres inédites à Charles Morice, éd. G. Zayed, Paris, Nizet, I969, p. 88 : «Veuillez appuyer sur mon côté inventif, narratif plutôt [....]. Il me semble que Crimen amoris, La Grâce, L'Impénitence finale, Don Juan pipé, Amoureuse du Diable [...] forment dans l'ensemble de ce que j'ai écrit un groupe assez important de choses à personnages pour être mentionné et apprécié.»

36. «Crimen amoris», «Don Juan pipé» et «L’impénitence finale». 
constitue la verve. Cet état non seulement existe à certains moments chez celui dont l'imagination engendre des œuvres, il subsiste imprimé dans ces œuvres elles-mêmes. On dira, sans hésiter, qu'un morceau est fait avec verve, qu'il y a de la verve dans telle tirade poétique, dans tel tableau, dans tel passage musical; que la verve de tel auteur est refroidie ou éteinte. [...] En général, les morceaux faits de verve se reconnaissent à l'entraînement qu'ils impriment à l'esprit du lecteur, de l'auditeur, du spectateur; ils sont accompagnés d'une grande facilité, à laquelle se joignent souvent des négligences [...]

La primauté de l'imagination; l'amplitude et la théâtralité impliquées par le mot «tirade», l'idée d'un élan, d'un emportement qui imprègnent le texte et se communiquent à l'esprit du lecteur, enfin la tolérance finalement accordée aux faiblesses de style, qui sont le corollaire inévitable d'une improvisation aisée - toutes ces caractéristiques de la verve telle qu'on la conçoit en I870 offrent une grille de lecture qui permet d'apprécier à leur juste valeur les poèmes que Verlaine compose, avec une étonnante célérité, lors de son séjour dans les prisons belges; en particulier ceux qu'il a placés dans la seconde partie du recueil Cellulairement et qui devaient préfigurer dans son esprit la tournure nouvelle que prendrait son œuvre à venir, à compter de son retour au sein de l'Église.

Pour finir, nous voudrions proposer une typologie de la verve poétique à partir du corpus que constituent ces textes. Trois caractéristiques principales s'en dégagent : c'est un discours en phase avec les valeurs socialement dominantes; s'y opère le transfert dans l'écriture d'une oralité et d'une sociabilité perdues; enfin c'est une parole autarcique, animée par l'auto-érotisme. Ce qui est frappant, et peut paraitre décevant, à la lecture du Verlaine seconde manière, c'est qu'il est passé de la dissidence au conformisme. La première manière de Verlaine, c'est-à-dire la poétique, parfois hermétique, de "la chanson grise» dans Romances sans paroles, si proche des "Derniers vers" de Rimbaud, allait de pair avec une révolte, parallèle à la Commune, contre l'ordre bourgeois du discours (la rhétorique des harangues publiques, l'éloquence académique, la clarté scolaire, la mise en ordre des matières...). La veine poétique murmurante et malingre des Romances sans paroles exprimait le rejet de la poésie parnassienne à la manière de Leconte de Lisle, qui représentait une sorte d'art officiel du Second Empire. Verlaine était alors dans la marginalité, aussi bien socialement (à cause de son homosexualité) que littérairement. Au contraire, la seconde partie de Cellulairement, qui constitue le récit de la réintégration de Verlaine au sein de l'Église (parallèlement à sa réhabilitation sociale par le châtiment judiciaire), marque aussi son entrée dans le cadre d'un discours socialement dominant : l'allégeance à la religion catholique et l'évolution politique vers le conservatisme voire la réaction (évolution que proclame l'épigraphe du recueil, 
empruntée à Joseph de Maistre ${ }^{37}$ ), coïncident de manière frappante avec ce qui se passe en France au début de la III ${ }^{\mathrm{e}}$ République, pendant la période de l'Ordre moral, sous la présidence de Mac-Mahon ${ }^{38}$ : le retour aux valeurs d'ordre, à l'autorité et au catholicisme; la volonté d'expiation et la soumission à une moralité austère. La verve, qui se targue volontiers de développer de brillants paradoxes, trouve en fait les conditions de possibilité de son effusion dans une complicité profonde avec les idées reçues et l'idéologie dominante.

Deuxième surprise concernant la verve : alors qu'on s'attend à ce qu'elle nécessite, pour prendre son essor, un public relativement nombreux assistant au spectacle de son déploiement improvisé, elle apparaît au contraire chez Verlaine comme parole solitaire et retranchée. Verlaine se convertit à la verve poétique au moment où il se retrouve complètement seul, en prison : mis au ban de la société et même des cénacles littéraires, éloigné de Rimbaud, officiellement séparé de Mathilde, il compose les poèmes de Cellulairement alors même que la possibilité de les publier paraît des plus incertaines, vu sa situation. Traditionnellement, la verve était liée à une parole en société. On le voit par exemple dans Sylvie de Nerval, où la verve apparaît dans le cadre du cercle, qui est l'avatar, à l'époque romantique, des salons de l'âge classique :

C'est ainsi que, sortant du thêâtre avec l'amère tristesse que laisse un songe évanoui, j'allais volontiers me joindre à la société d'un cercle où l'on soupait en grand nombre, et où toute mélancolie cédait devant la verve intarissable de quelques esprits éclatants, vifs, orageux, sublimes parfois, — tels qu'il s'en est trouvé toujours dans les époques de rénovation et de décadence, et dont les discussions se haussaient à ce point, que les plus timides d'entre nous allaient voir parfois aux fenêtres si les Huns, les Turcomans ou les Cosaques n’arrivaient pas enfin pour couper court à ces arguments de rhéteurs et de sophistes ${ }^{39}$.

Il y aurait ainsi une verve classique, qui durerait jusqu'au romantisme et resterait associée à l'oralité grâce à l'existence de lieux de réunion qui offraient une scène à sa performance; et une verve moderne, ayant son origine au début de la décennie I870-I880, et liée à l'isolement définitif de l'écrivain qui ne trouve même plus d'insertion possible dans des cénacles littéraires devenus parodiques, comme les Vilains Bonshommes, les Zutistes, les Hydropathes, etc.

37. Cette épigraphe n’apparaît pas dans le manuscrit principal du recueil, mais dans la Lettre à Ernest Delahaye du 7 mai I875 : «Dans les fers! Voyez un peu le poëte!) (J. de Maistre.)» (Correspondance générale I, ouvr. cité, p. 398.)

38. La concomitance des trajectoires individuelle de Verlaine et collective de la III ${ }^{e}$ République à ses débuts est remarquable : l'Ordre moral correspond plus précisément au premier ministère de Broglie, de mai I873 à mai I874, précisément le moment où Verlaine revient au catholicisme et abjure ses " égarements" passés.

39. Nerval, Sylvie, Paris, Librairie générale française, coll. «Le Livre de poche Libretti », I999, p. 29 . 
La verve se transfère de l'oral à l'écrit sous l'effet de l'isolement du poète moderne, héritier de Baudelaire. Elle devient un style consistant à mimer par des procédés d'écriture l'allure d'une parole encouragée à se déverser par la présence d'un public qui soutient l'orateur.

Enfin, la verve semble manifester un fort investissement libidinal de la parole : l'énergie de ce discours jaillissant traduit l'excitation du locuteur, censé communiquer à son public le plaisir qu'il prend à pérorer. Mais cette jouissance, que l'on décèle dans la définition de la verve sous le terme convenu d' 'enthousiasme», est placée sous le signe de l'auto-érotisme. Apparaissant dans l'œuvre verlainienne au moment où l'écrivain est condamné à l'abstinence par sa condition de prisonnier, la verve semble procéder d'une dérivation de la pulsion érotique dans la parole, qui se met à jouir de son propre débordement. La verve moderne serait donc une «machine célibataire». Cette hypothèse permet en tout cas d'éclairer les lacunes du manuscrit principal de Cellulairement: comme l'a montré Olivier Bivort ${ }^{40}$, deux poèmes faisant initialement partie du recueil en ont été détachés, et leur élimination explique les lacunes du manuscrit aux endroits où il a été découpé : «À ma femme en lui envoyant une pensée » était situé après "Réversibilités", dans la première partie du recueil; et "À qui de droit» après "L’Art poëtique», au début de la seconde partie. Un troisième poème, "Bouquet à Marie», fait aussi incontestablement partie du projet éditorial de Cellulairement, quoiqu'on ne retrouve pas sa place dans le manuscrit principal. Or, ces trois poèmes supprimés du recueil ont un point commun : ce sont ceux où Verlaine évoquait explicitement le fait qu'il a été marié. Sa femme est la destinatrice des deux premiers, et leur mariage est évoqué explicitement à la neuvième strophe de "Bouquet à Marie», alors qu'il est passé sous silence dans l'ensemble des poèmes finalement retenus pour le recueil ${ }^{41}$ (resté inédit, certes, mais le manuscrit à partir duquel nous l'étudions était destiné à l'imprimeur, comme le montre entre autres choses le fait que Verlaine a souligné d'un double trait toutes les lettres initiales devant être imprimées en majuscule). Tout se passe comme si celui qui se donnera faussement pour veuf dans ses mémoires avait voulu se faire passer pour célibataire dans le recueil où, concomitamment, il expérimente la verve poétique.

Il n'y a aucune raison objective de récuser le témoignage de Verlaine luimême sur la genèse des poèmes de Cellulairement : ces vers ont, semble-t-il, bien été composés en prison, à partir de juillet I873 et à un rythme rapide. Au

40. Ouvr. cité, p. 72-73.

4I. Il est bien question de «ma compagne» dans «Vieux Coppées III », mais cette expression n'implique pas stricto sensu la condition d'épouse. 
fond, c'est la productivité élevée que suppose cette chronologie de la composition des textes qui a fait révoquer en doute et la datation des poèmes par leur auteur et le témoignage ultérieur de ses mémoires. D'autant qu'une telle fécondité contraste avec la parcimonie de la production antérieure, faite de recueils courts voire diaphanes (Fêtes galantes, Romances sans paroles) et espacés dans le temps. Mais cette soudaine fécondité s'explique par l'adoption, délibérée de la part de Verlaine, d'une nouvelle pratique de l'écriture versifiée, fondée sur la verve poétique : une écriture abondante, rapide, ne craignant pas une certaine négligence dès lors que sa priorité est de communiquer la force d'entraînement de l'inspiration dont elle procède. Cette forme d'écriture poétique se veut populaire, à la fois parce qu'elle emprunte des registres d'expression familiers, proches de l'oral spontané, et parce qu'elle aspire à une large diffusion éditoriale. (Sur ce point, Verlaine aura connu une complète déconvenue, mais qui n'enlève rien au fait qu'il a désiré ou rêvé un succès d'édition qui lui aurait permis de vivre de sa plume, comme en témoigne la lettre du 28 septembre I873 à Edmond Lepelletier : «J'ai mille projets littéraires : du théâtre surtout, car j'entends, dès ma sortie, me remuer jusqu'à ce que je gagne sérieusement de l'argent avec "ma plume ${ }^{42}$ ".») Mais la conception de la poésie qui rend possible la verve poétique date de l'époque romantique, où un recueil de poèmes pouvait encore être un succès de librairie. Au début des années I870, cette conception est en train d'être rendue caduque par l'évolution du marché éditorial, qui va faire de la poésie, à l'époque symboliste, une «niche» vouée aux tirages limités et aux éditions de luxe. Cette évolution du genre poétique s'accompagne d'une théorisation, principalement par Mallarmé, qui va imposer une nouvelle conception de la poésie foncièrement incompatible avec la notion même de verve. Dans "Crise de vers", notamment, Mallarmé définit la poésie comme une langue séparée du langage courant, langue mystérieuse et quintessenciée, nécessitant du lecteur un effort de déchiffrement. La verve poétique implique au contraire que la parole du poète soit de plain-pied avec «l'universel reportage dont, la littérature exceptée, participe tout entre les genres d'écrits contemporains ${ }^{43} »$. Verlaine d'ailleurs emprunte beaucoup à la langue des journaux dans les poèmes de Cellulairement, particulièrement dans les «Vieux Coppées». C’est ainsi que le projet esthétique qui préside à la composition de Cellulairement, et qui apparaît dans la structure même de ce recueil, a été rendu incompréhensible par la situation particulière de Verlaine, dont les travaux des années I872-I875 (Romances sans paroles et les poèmes de

42. Correspondance générale I, ouvr. cité, p. 35I.

43. S. Mallarmé, Igitur. Divagations. Un coup de dés, éd. B. Marchal, Paris, Gallimard, coll. "Poésie/Gallimard», 2003, p. 259. 
Cellulairement, en particulier "L’Art poëtique») ne seront découverts par le public lettré qu'une décennie plus tard, grâce à leur publication en revue, au moment de l'émergence du courant symboliste. L'annexion a posteriori de Verlaine par les symbolistes (à laquelle lui-même s'opposait avec mauvaise grâce et mauvaise humeur, comme en atteste son interview par Jules Huret ${ }^{44}$ ) a contribué à sa célébrité et lui a permis de passer à la postérité (notamment grâce à son élection au titre de "prince des poètes" en I894), mais au prix d'un profond malentendu esthétique, qui a fait méjuger quasiment toute sa production ultérieure au tournant de I873-I874, c'est-à-dire la "seconde manière» de Verlaine, dont la notion de "verve poétique» est peut-être le sésame.

44. "M. Paul Verlaine», dans Enquête sur l'évolution littéraire [I89I], éd. D. Grojnowski, Vanves, Éditions Thot, I984, p. 80-85. 